\title{
TopBPI contributes to the chemoresistance in non-small cell lung cancer through upregulation of $\mathrm{p} 53$
}

This article was published in the following Dove Press journal:

Drug Design, Development and Therapy

23 September 2016

Number of times this article has been viewed

\author{
Yinxiang $L v^{1, *}$ \\ Yanan Huo ${ }^{2, *}$ \\ Xican $\mathrm{Yu}^{\prime}$ \\ Rongrong $\mathrm{Liu}^{3}$ \\ Shufen Zhang ${ }^{3}$ \\ Xiaoxiao Zheng ${ }^{3}$ \\ Xianning Zhang ${ }^{3}$ \\ 'Department of Oncology, Xinchang \\ People's Hospital, Shaoxing, ${ }^{2}$ Eye \\ Centre, Second Affiliated Hospital \\ of Zhejiang University, School of \\ Medicine, Hangzhou, ${ }^{3}$ Department of \\ Cell Biology and Medical Genetics, \\ Research Center of Molecular \\ Medicine, National Education Base \\ for Basic Medical Sciences, Institute \\ of Cell Biology, Zhejiang University \\ School of Medicine, Hangzhou, \\ People's Republic of China
}

*These authors contributed equally to this work
Correspondence: Yinxiang Lv Department of Oncology, Xinchang People's Hospital, Gushan Middle Road I 17, Shaoxing 3/2500, Zhejiang Province, People's Republic of China Emailyinx12015@I26.com

\begin{abstract}
Resistance to chemotherapeutic drugs is a major obstacle in non-small cell lung cancer (NSCLC) therapy. The molecular determinants of NSCLC resistance to doxorubicin are unknown. In the present study, we investigated whether topoisomerase II $\beta$ binding protein 1 (TopBP1) was involved in the chemoresistance to doxorubicin in NSCLC cancer. We found that p53-deficient lung cancer cells (NCI-H1299) displayed the greatest resistance to doxorubicin compared with NCI-H358, A549, and HCC827 cells with p53 expression. The expression of TopBP1 was significantly higher in NCI-H1299 cells than the other three tumor cell lines. In addition, TopBP1 knockdown with specific small interfering RNA in NCI-H1299 cells enhanced the doxorubicin chemosensitivity and decreased the expression of $\mathrm{p} 53$ in the presence of doxorubicin. After doxorubicin administration, co-immunoprecipitation assay showed that TopBP1 promoted the expression of p53 in NCI-H1299 cells. These results for the first time demonstrated that TopBP1 plays an important role in NSCLC chemoresistance via upregulation of $\mathrm{p} 53$. Therefore, inhibition of TopBP1, in combination with chemotherapy, may represent a novel strategy for the treatment of chemotherapy-resistant NSCLC.
\end{abstract}

Keywords: non-small cell lung cancer, drug resistance, TopBP1, p53

\section{Introduction}

Lung cancer is the leading cause of cancer-related death worldwide; $80 \%$ of lung cancers are non-small cell lung cancer (NSCLC) with poor therapeutic efficacy when diagnosed. ${ }^{1,2}$ It is estimated that approximately $40 \%$ of patients with NSCLC present with advancedstage disease for which 5-year survival rates are in the region of $2 \% .{ }^{3}$ Currently, platinumbased regimens are the mainstay of lung cancer therapy, and chemotherapy serves as one of the important adjuvant therapies for its treatment. ${ }^{4}$ However, drug resistance to conventional chemotherapeutics has become a major handicap in the success of NSCLC chemotherapy. 5,6 Thus, it is imperative to develop novel therapeutic strategies that may enhance tumor cell response to anticancer drugs. Recently, studies have begun to investigate the molecular mechanism of NSCLC and identified various novel targeted agents, such as epidermal growth factor receptor tyrosine kinase inhibitors which exhibit greater efficacy than chemotherapy in patients with epidermal growth factor receptor-mutated tumors. ${ }^{7}$ Despite the great progresses achieved in cancer therapy, the molecular mechanism of lung cancer pathogenesis and chemoresistance still remains elusive.

Topoisomerase II $\beta$ binding protein 1 (TopBP1) was identified as an interacting partner for topoisomerase II $\beta .{ }^{8,9}$ It contains nine BRCA1 carboxyl-terminal domains and functions in DNA damage response, DNA checkpoint activation, replication, and 
regulation of transcription. ${ }^{10-13}$ TopBP1 also interacts several transcriptional factors, such as p53, ${ }^{14} \mathrm{E} 2 \mathrm{~F} 1,{ }^{15,16} \mathrm{Miz} 1,{ }^{17}$ and 53BP1. ${ }^{18}$ Regulation of p53 by TopBP1 plays an important role in the regulation of proapoptotic activity and cell cycle transition. ${ }^{19}$ It is reported that TopBP1 is frequently overexpressed in cancer and inactivates p53 functions. ${ }^{19}$ In the current study, we aimed to explore the biological role of TopBP1 in chemoresistance along with the molecular mechanism underling these effects.

\section{Materials and methods Cell culture and reagents}

Human lung cancer cell lines NCI-H358, A549, NCI-H1299, and $\mathrm{HCC} 827$ were purchased from the American Type Culture Collection (ATCC) (Manassas, VA, USA) and cultured in Dulbecco's Modified Eagle's Medium (Gibco, Carlsbad, CA, USA) supplemented with 10\% FBS and 1\% penicillin/streptomycin. All cells were maintained at $37^{\circ} \mathrm{C}$ in $5 \% \mathrm{CO}_{2}$ incubator. Doxorubicin was purchased from Sigma-Aldrich (St Louis, MO, USA). The TopBP1 small interfering RNA (siRNA) and negative control siRNA were purchased from Santa Cruz Biotechnology (Santa Cruz, CA, USA). As clinical samples or animals were not used in this study ethical approval was not required by the institutional review board.

\section{CCK-8 assay}

Cancer cells or siRNA-transfected cancer cells were seeded onto 96-well plates at 3,000 cells/well. The medium was replaced with the corresponding serum-free medium for 24 hours to synchronize the cell cycle, and then the serum-free medium was replaced with complete medium containing the drugs at the indicated concentrations. Then, $10 \mu \mathrm{L} /$ well CCK-8 solution (Dojindo, Kumamoto, Japan) was added, the plates incubated for 3 hours, and absorbance was measured at $450 \mathrm{~nm}$ using an MRX II microplate reader (Dynex, Chantilly, VA, USA).

\section{EdU incorporation assay}

Cell proliferation was calculated using EdU incorporation assay. Measurement of the inhibitive rate of cell proliferation was carried out using a Click-iTEdU Imaging Kit (Thermo Fisher Scientific, Waltham, MA, USA) according to the manufacture's protocol.

\section{Flow cytometry analysis}

Tumor cells were exposed to doxorubicin and p53 siRNA alone or in combination. After treatment for 48 hours, cells were trypsinized and centrifuged rpm for 5 minutes and the pellet washed twice with phosphate-buffered saline (PBS).
Cells were resuspended and then washed with PBS three times. Apoptosis cells were detected with annexin V-FITC/PI according to the protocol of Annexin V-FITC cell Apoptosis Detection Kit (Sigma-Aldrich Co., St Louis, MO, USA).

\section{siRNA transfection}

Lung cancer cells were seeded to achieve 30\%-50\% confluency on the day of transfection. Cells were transfected with TopBP1 siRNA, p53 siRNA, or negative control siRNA using Lipofectamine 2000 (Thermo Fisher Scientific, Waltham, MA, USA) according to the manufacturer's instruction. The transfection medium (Opti-MEM; Gibco) was replaced with complete medium 12 hours after transfection for further analysis.

\section{Western blot analysis}

Cells were lysed in $50 \mu \mathrm{L}$ cell lysis buffer (Cell Signaling, Danvers, MA, USA). The protein concentration was quantified using the BCA Protein Kit (Thermo, Rockford, IL, USA). Cell lysates were separated by $10 \%$ SDS-PAGE and proteins were transferred to polyvinylidenedifluoride membranes (Merck Millipore, Billerica, MA, USA). The membranes were then incubated with anti-TopBP1 or anti-p53 antibodies (Abcam, Cambridge, MA, USA) at $4^{\circ} \mathrm{C}$ overnight. The membranes were washed three times with $\mathrm{TBS} / \mathrm{T}$ and then incubated with the appropriate HRP-conjugated secondary antibodies (Abcam) for 1 hour at room temperature. Protein expression was detected by chemiluminescence (GE Healthcare Bio-Sciences Corp., Piscataway, NJ, USA).

\section{Co-immunoprecipitation}

Cells were harvested and lysed in $50 \mu \mathrm{L}$ cell lysis buffer (Cell Signaling) after treatment with doxorubicin for 48 hours. An aliquot of the cell lysates was lysed with SDS lysis buffer, and the rest of the cell lysates were incubated with appropriate antibodies or beads for 24 hours at $4^{\circ} \mathrm{C}$. Immunoprecipitates were fractionated by SDS-PAGE and electrotransferred to the polyvinylidenedifluoride membranes (Merck Millipore). The specific signals were detected with anti-TopBP1 or anti-p53 antibodies (Abcam). Protein expression was detected using chemiluminescence (GE Healthcare Bio-Sciences Corp.).

\section{Statistical analysis}

Each experiment was performed in triplicate and repeated at least three times. All the data were presented as mean \pm standard deviation and treated for statistical analysis by Statistical Product and Service Solutions (SPSS) 16.0 (IBM, Armonk, NY, USA). Comparison between groups was made using one-way ANOVA and a statistically significant difference was defined as $P<0.05$. 


\section{Results}

\section{Different doxorubicin sensitivity and} TopBPI expression in lung cancer cells

Firstly, CCK-8 assay we performed to examine cell viability of different lung cancer cell lines (NCI-H358, A549, NCI-H1299, and HCC827) after incubation with doxorubicin for 48 and 72 hours. It was found that the doxorubicin sensitivity varied among cell lines (Figure 1A-D). As shown in Table 1, the
$\mathrm{IC}_{50}$ values at 48 or 72 hours were significantly higher in NCI-H1299 cells compared with the other three cell lines. In addition, the protein expression of TopBP1 was detected in different cell lines by Western blot. We found that TopBP1 expression was significantly higher in NCI-H1299 cells compared with three other cell lines (Figure 1E). Furthermore, EdU incorporation assay was carried out to measure the proliferation of lung cancer cells. Results showed that the inhibitive effect of

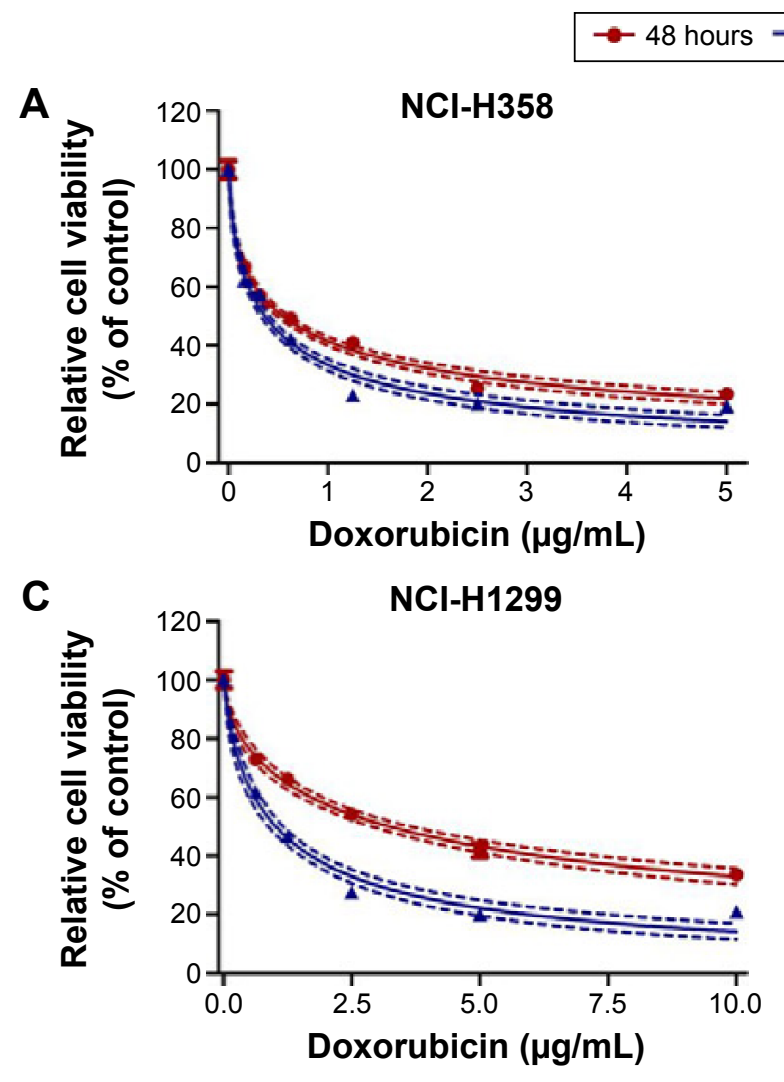

72 hours
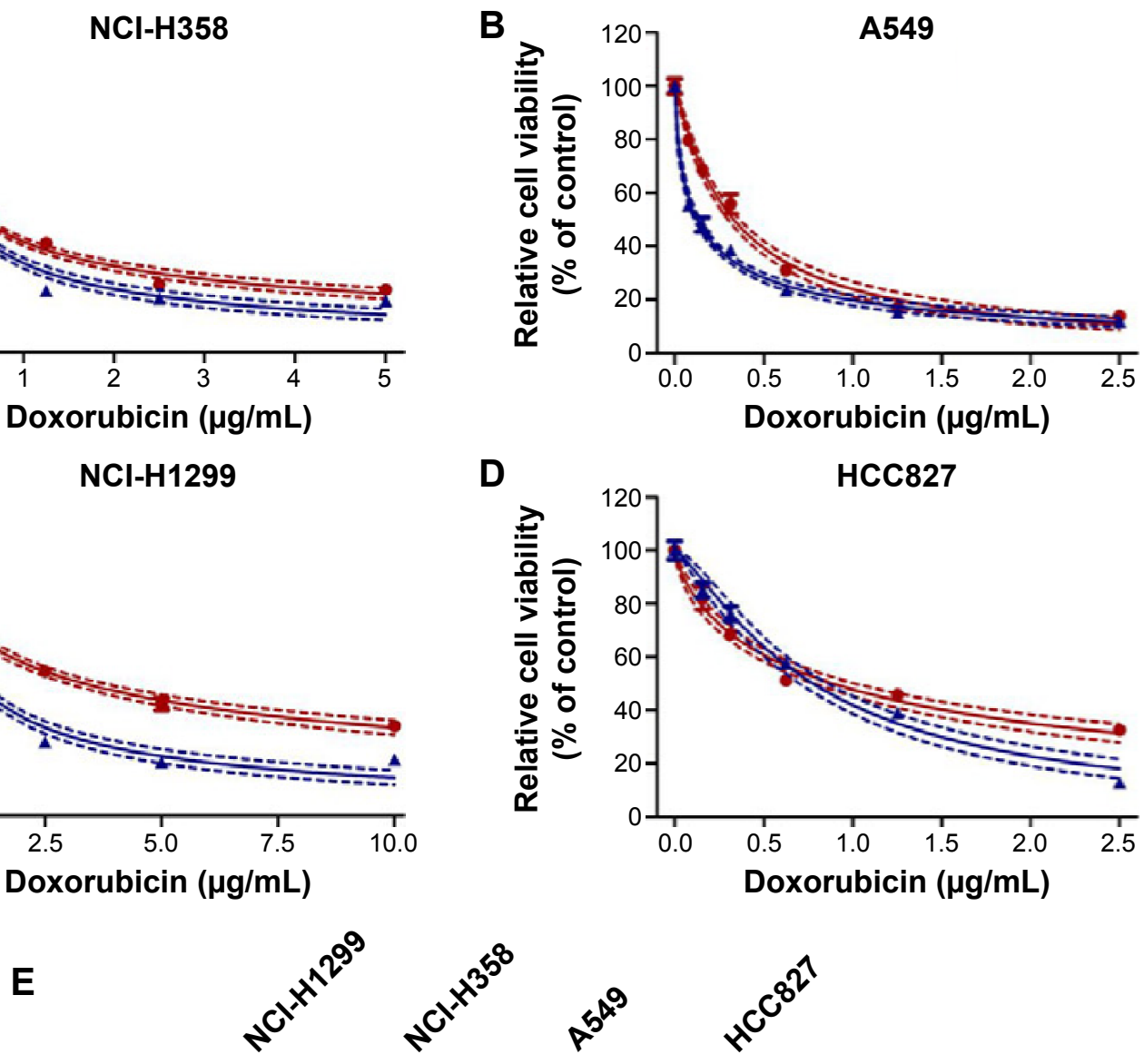

TopBP1

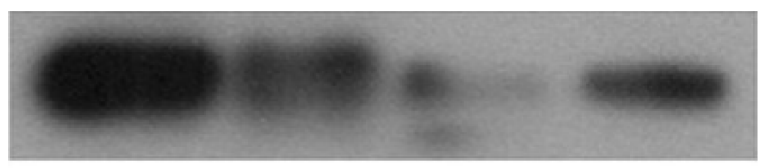

p53

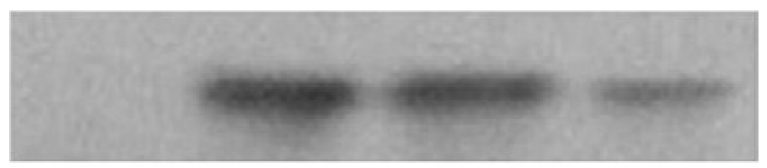

GAPDH

Figure I Different doxorubicin sensitivity and topoisomerase II $\beta$ binding protein I (TopBPI) expression in lung cancer cells.

Notes: Lung cancer cells were incubated with different concentrations of doxorubicin for 48 or 72 hours. CCK-8 assay was applied to measure the cell viability of different lung cancer including NCl-H358 (A), A549 (B), NCl-HI299 (C), and HCC827 (D). Western blot was used to detect the protein expression of TopBPI in four cancer cell lines (E). 
Table I Determination of doxorubicin $\mathrm{IC}_{50}$ in different tumor cell lines

\begin{tabular}{lll}
\hline $\mathrm{IC}_{50}(\mu \mathrm{g} / \mathrm{mL})$ & $\mathbf{4 8}$ hours & $\mathbf{7 2}$ hours \\
\hline $\mathrm{NCl}-\mathrm{H} 358$ & 0.5409 & 0.3677 \\
$\mathrm{~A} 549$ & 0.3304 & 0.1263 \\
$\mathrm{HCC} 827$ & 0.8896 & 0.777 \\
$\mathrm{NCl}-\mathrm{HI} 299$ & 3.423 & 1.030 \\
\hline
\end{tabular}

Abbreviation: $\mathrm{IC}_{50}, 50 \%$ inhibitory concentration.

doxorubicin was reduced in NCI-H1299 cells compared with that in NCI-H358, A549, and HCC827 cells (Figure 2A-D). These results implied that the TopBP1 might be associated with the drug resistance to doxorubicin in NSCLC cells.
TopBPI was involved in the chemoresistance of NSCLC cells

In order to confirm whether TopBP1 was involved in the tumor cells chemoresistance, TopBP1 siRNA was transfected into lung cancer cells to interfere with TopBP1 expression. Western blot showed that the TopBP1 expression was obviously decreased in four cancer cell lines after transfection with TopBP1 siRNA (Figure 3A). Addition of doxorubicin increased the p53 expression in NCI-H358, A549, and HCC 827 cells and, interestingly, induced the expression of p53 in NCI-H1299 cells (Figure 3A). Moreover, we found
A

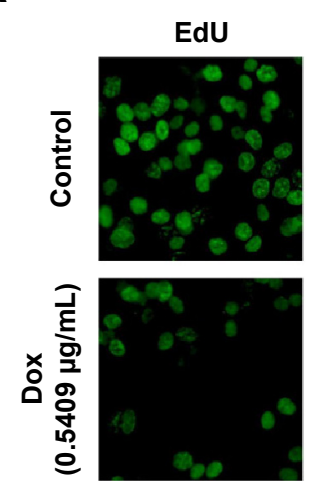

B

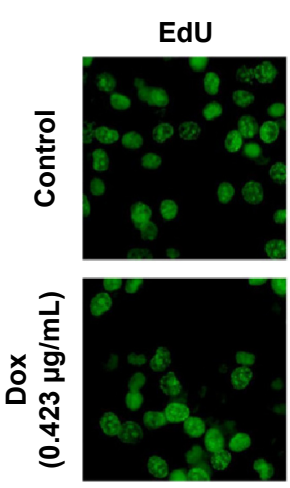

C

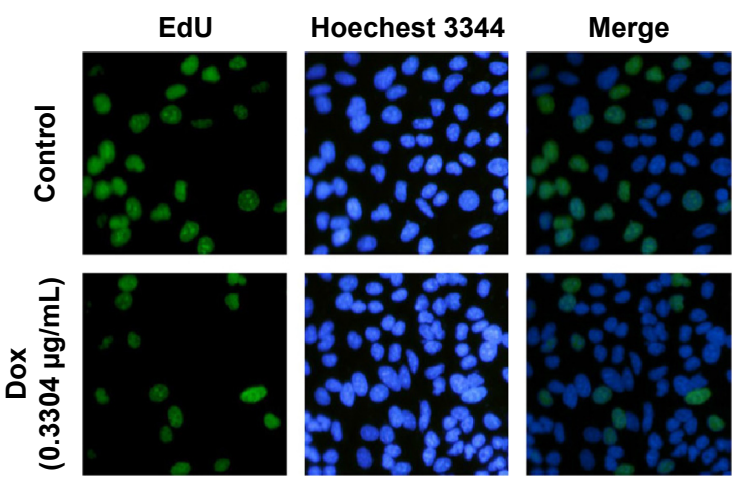

NCI-H358
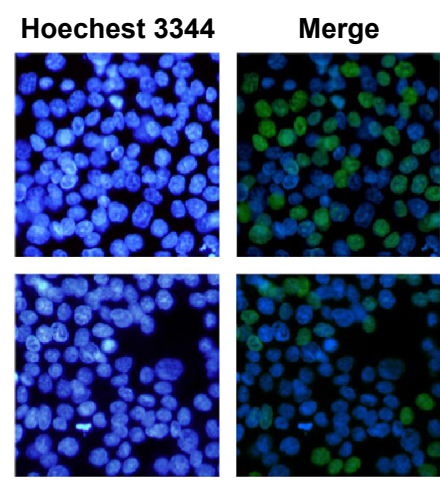

NCl-H1299

Hoechest 3344
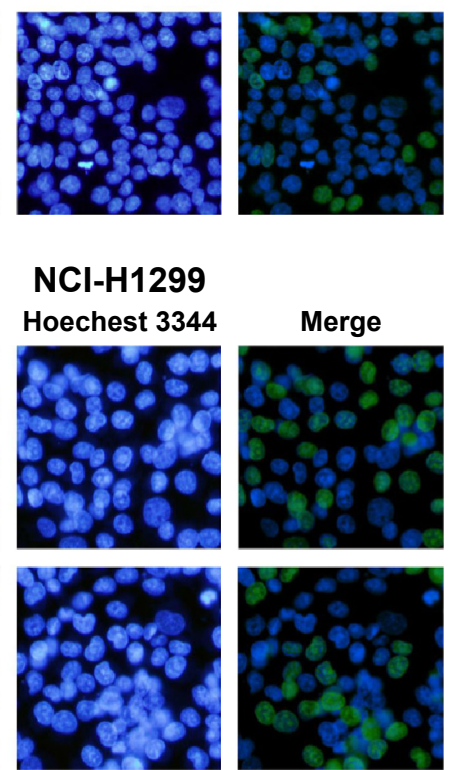

Merge
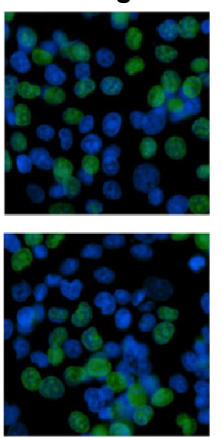

A549

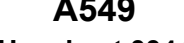

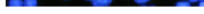
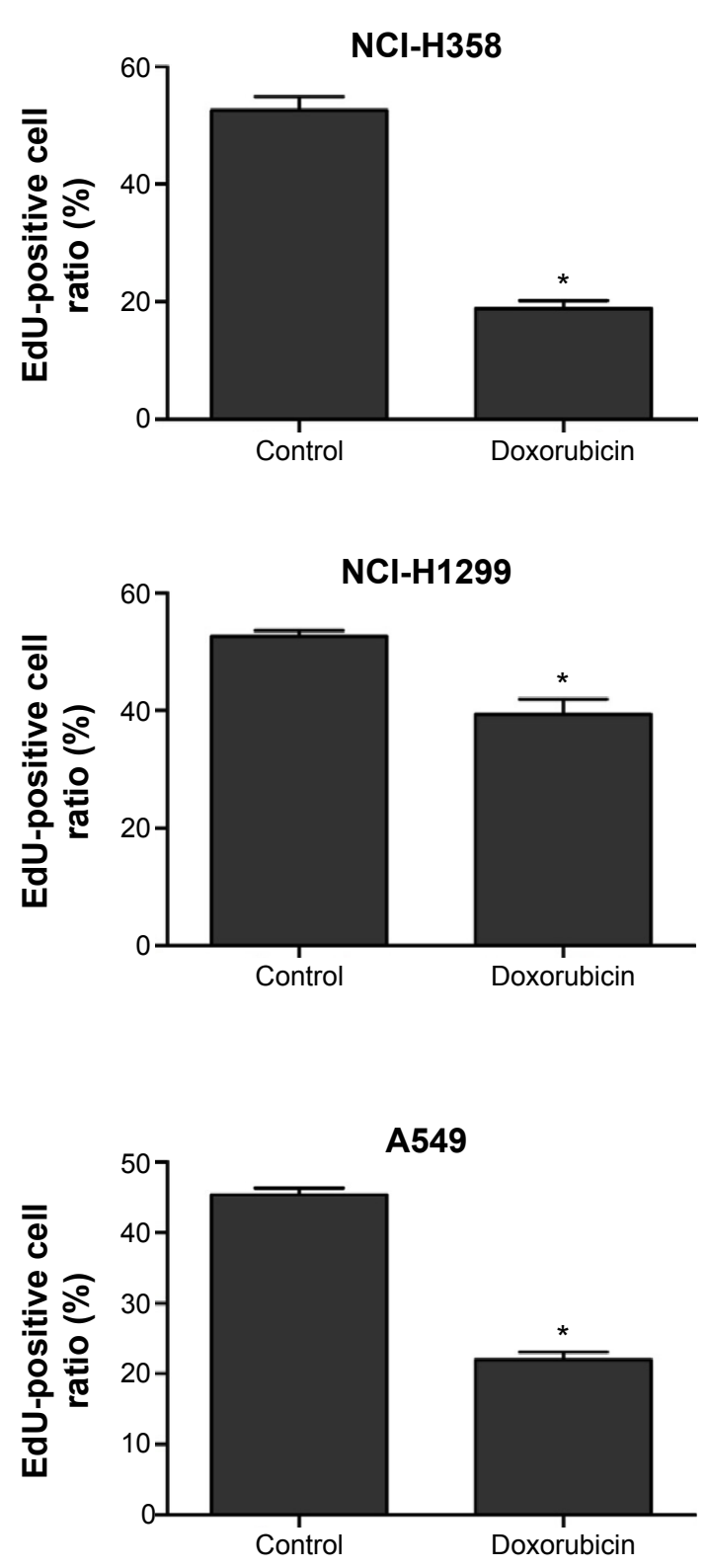

Figure 2 (Continued) 
D
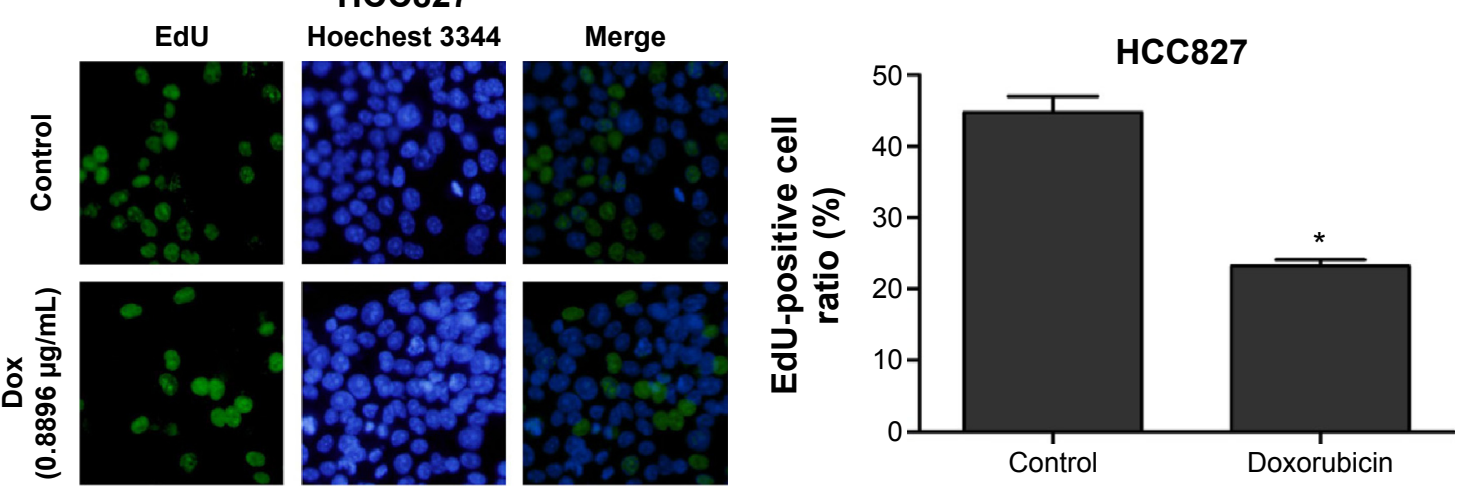

Figure 2 Measurement of cell proliferation in lung cancer cells.

Notes: Lung cancer cells were incubated with or without doxorubicin (Dox) for 48 hours. EdU incorporation assay was performed to measure the proliferation of $\mathrm{NCl}-\mathrm{H} 358$ (A), NCl-HI 299 (B), A549 (C), and HCC827 (D) cells. *P<0.05.

that $\mathrm{p} 53$ siRNA-transfected NCI-H1299 cells became more sensitive to doxorubicin, while downregulation of p53 in other three cell lines led to no significant changes in their cellular responses to doxorubicin treatment (Figure S1). CCK-8 assay demonstrated that TopBP1 knockdown increased the sensitivity of NCI-H1299 (Figure 3B), NCI-H358, A549, and HCC827 cells (Figure S2) to doxorubicin. These data suggested that TopBP1 was involved in NSCLC cell sensitivity to doxorubicin.

\section{Downregulation of TopBPI sensitized $\mathrm{NCl}-\mathrm{HI} 299$ cells to doxorubicin}

We further performed EdU incorporation assay to measure the proliferation of lung cancer cells after transfection with TopBP1 siRNA. Results showed that TopBP1 downregulation led to no significant differences in the proliferation of NCI-H358 (Figure 4A), A549 (Figure 4C), and HCC827 (Figure 4D) cells in the presence of doxorubicin. However, the inhibitive effect of doxorubicin was increased in
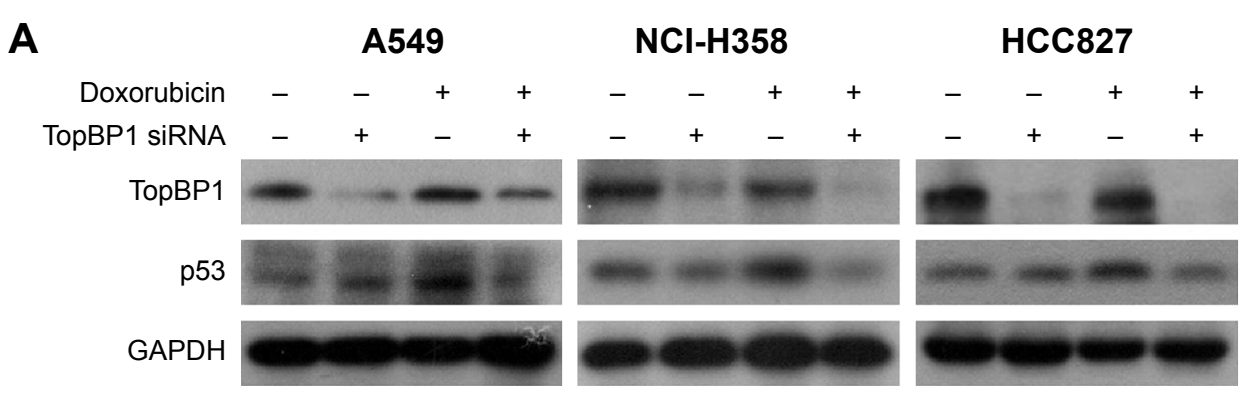

NCl-H1299

B

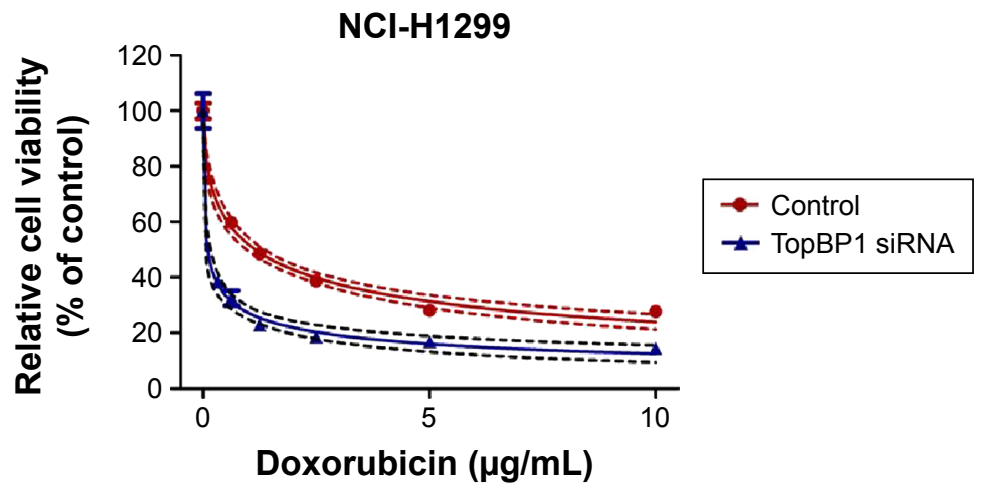

Figure 3 TopBPI was involved in the chemoresistance of tumor cells.

Notes: TopBPI siRNA was transfected into different lung cancer cells and Western blot was applied to detect the protein levels of TopBPI and p53 in tumor cells with or without doxorubicin treatment (A). After transfection, the chemosensitivity of $\mathrm{NCl}-\mathrm{HI} 299$ cells to doxorubicin was measured using CCK-8 methods (B).

Abbreviations: TopBPI, topoisomerase II $\beta$ binding protein I; siRNA, small interfering RNA. 
A
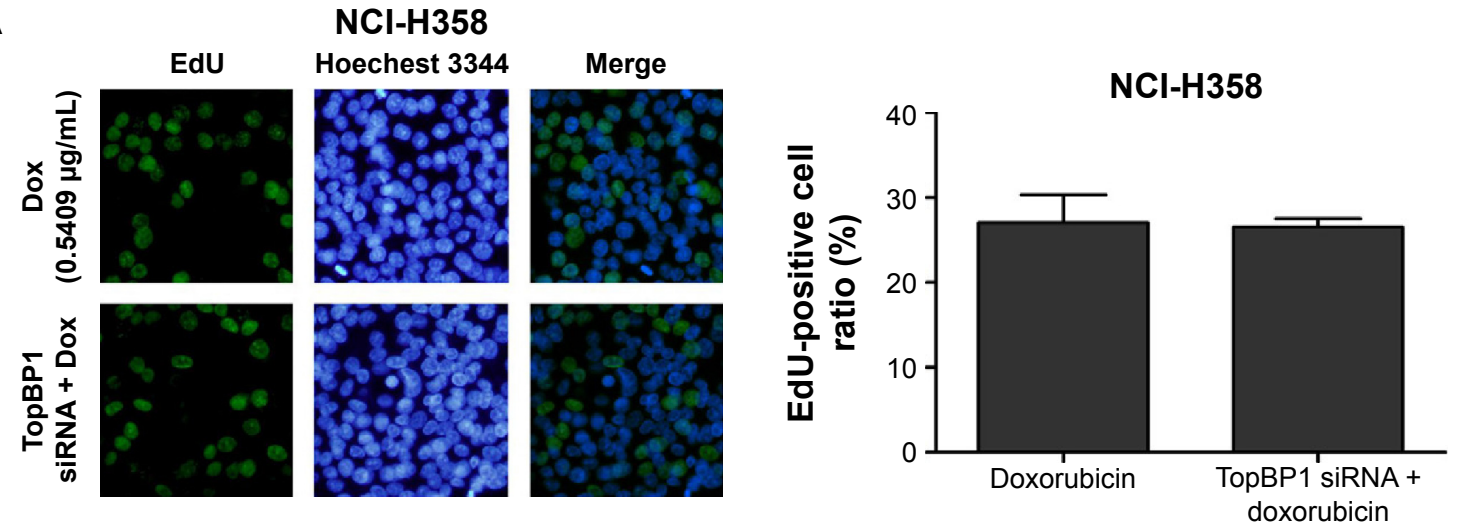

B
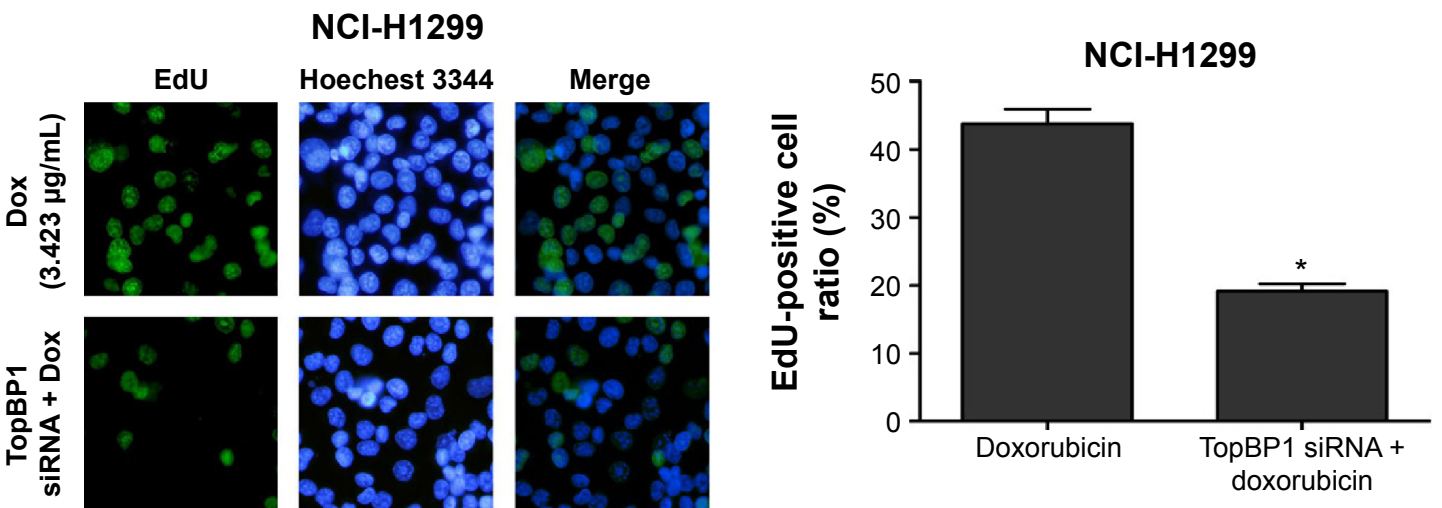

C
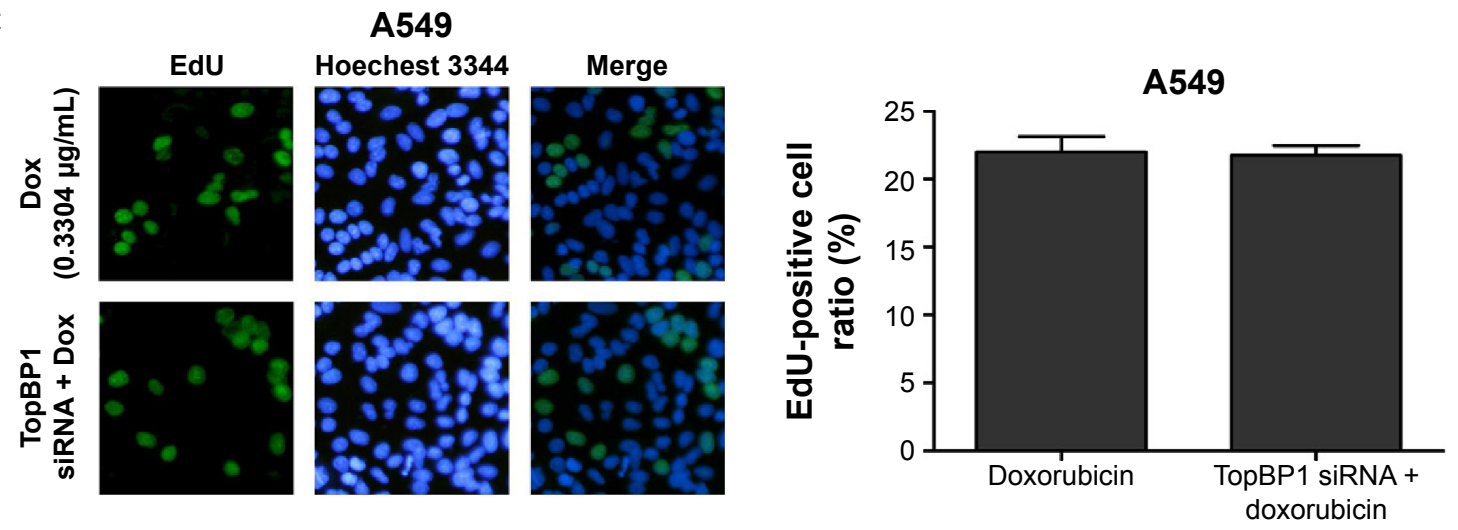

D
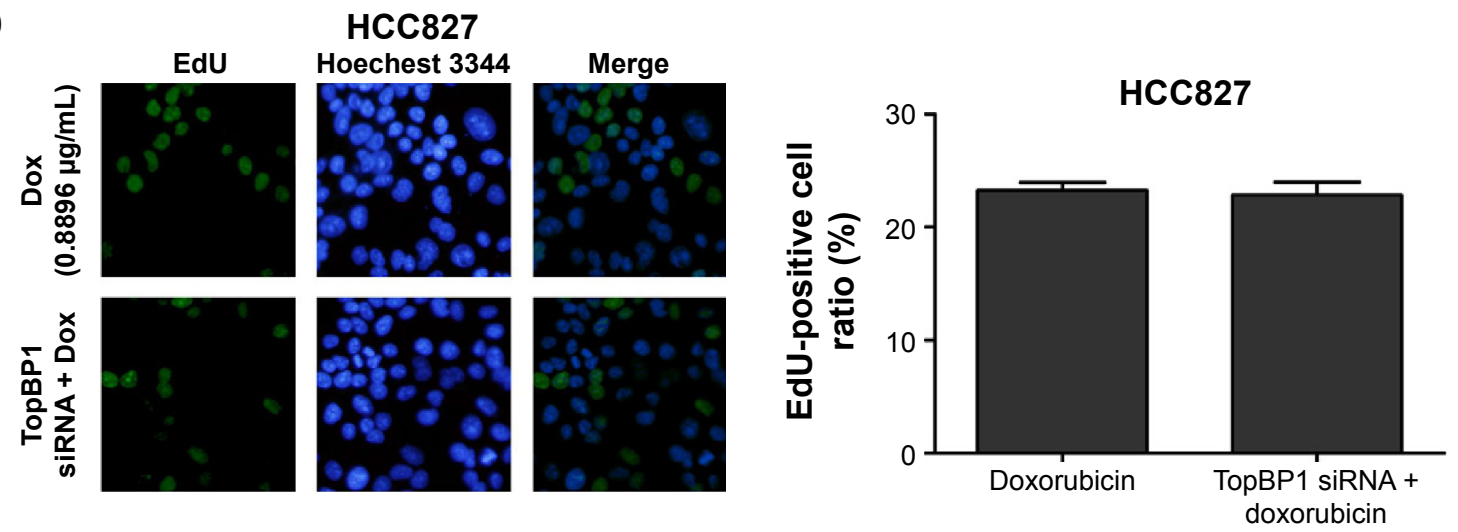

Figure 4 Measurement of cell proliferation in TopBPI siRNA-transfected lung cancer cells.

Notes: Lung cancer cells were transfected with TopBPI siRNA or control siRNA in the presence of doxorubicin (Dox). EdU incorporation assay was performed to measure the proliferation of $\mathrm{NCl}-\mathrm{H} 358$ (A), $\mathrm{NCl}-\mathrm{HI} 299$ (B), A549 (C), and $\mathrm{HCC} 827$ (D) cells. $* \mathrm{P}<0.05$.

Abbreviations: TopBPI, topoisomerase $I / \beta$ binding protein I; siRNA, small interfering RNA. 
TopBP1 siRNA-transfected NCI-H1299 cells compared with doxorubicin treatment alone (Figure 4B).

\section{Doxorubicin-induced p53 was mediated by TopBPI in NSCLC cells}

Finally, co-immunoprecipitation was performed to investigate the relationship between TopBP1 and doxorubicininduced $\mathrm{p} 53$. It was demonstrated that TopBP1 was involved in the p53 induction after doxorubicin administration in NCI-H1299 cells (Figure 5A). However, there are little changes in p53 levels in NCI-H358, A549, and HCC827 cells (Figure 5B). These results showed that the drug resistance was regulated by TopBP1-dependent $\mathrm{p} 53$ induction by doxorubicin in NCI-H1299 cells.

\section{Discussion}

Currently, chemotherapy serves as an important component of postoperative treatment for NSCLC. However, development of drug resistance to the conventional chemotherapeutics has become a major challenge to successful chemotherapy. ${ }^{20,21}$ Thus, it is urgent that we find novel therapeutic targets involved in the acquisition of drug resistance. In this study, we demonstrated that TopBP1 modulated the chemoresistance to doxorubicin in NSCLC cells. It is noteworthy to mention that, to our knowledge, this study was the first to demonstrate the therapeutic potential of TopBP1 in lung cancer.

TopBP1 has been shown to be involved in DNA damage response, chromosome replication, and transcription. ${ }^{10-13}$ Depletion of TopBP1 led to embryonic lethality at the peri-implantation stage and cellular senescence in primary cells. ${ }^{22,23}$ In breast cancer, Liu et al observed overexpression of TopBP1 in tumor tissues and demonstrated that those patients with higher expression of TopBP1 had shorter overall survival time than those without overexpression of TopBP1. ${ }^{14}$ In a recent study, the expression of TopBP1 was found to be upregulated in cancer cells and depletion of TopBP1 enhanced the sensitivity to radiation in radio-resistant lung cancer cells. ${ }^{24}$ Our study found that NCI-H1299 cells exhibited the lowest sensitivity to doxorubicin among four lung cancer cell lines. Interestingly, Western blot analysis showed that the protein level of TopBP1 was higher in NCIH1299 cells compared with NCI-H358, A549, and HCC827 cells. Thus, it was hypothesized that enhanced expression of TopBP1 might be involved in the chemoresistance to doxorubicin. To test such a hypothesis, the TopBP1 expression was decreased via transfection of TopBP1 siRNA into NCI-H1299 cells. Consequently, TopBP1 knockdown in
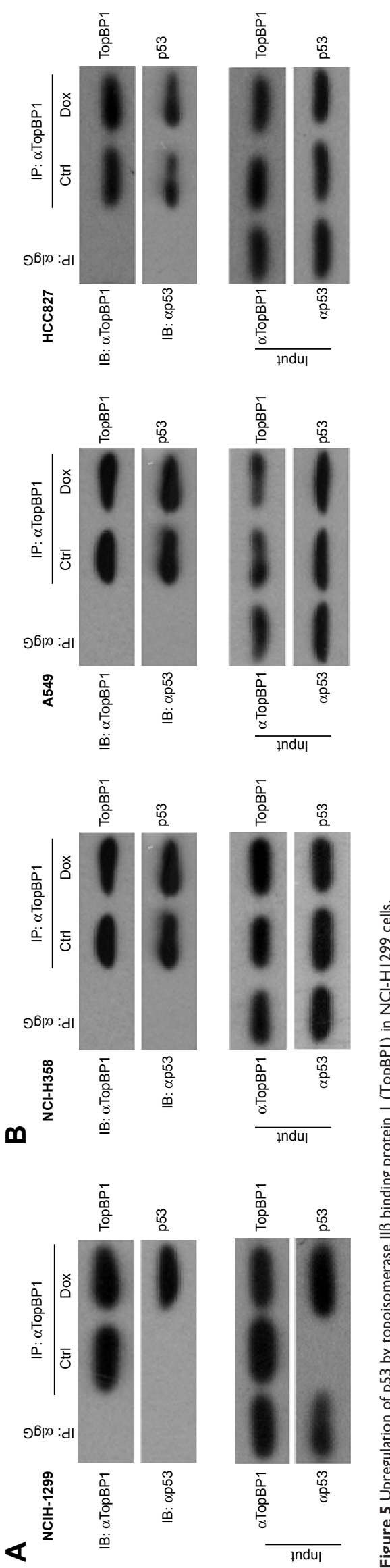
NCI-H1299 cells increased the sensitivity to doxorubicin. These results suggested that TopBP1 was a critical modulator in the acquired chemoresistance of lung cancer cells.

The tumor suppressor p53 is a transcription factor that responds to various types of cellular stress, such as oncogene activation and genotoxic drug-induced DNA damage..$^{25,26}$ p53 regulates a variety of cellular functions, including DNA repair, cell cycle arrest, and apoptosis. ${ }^{27-29}$ Abnormal expression of p53 is found in nearly all types of cancers, and p53 mutations are associated with resistance to chemotherapeutics and poor patient prognosis..$^{30-33}$ In our study, the p53-negative NCI-H1299 cells displayed more doxorubicin resistance than three other cell lines with p53 expression. In order to explore the role of p53 in TopBP1-modulated chemosensitivity, we examined TopBP1 and p53 expression with or without doxorubicin treatment. Our data showed that doxorubicin administration resulted in upregulation of p53 in NCI-H358, A549, and HCC827 cells. Interestingly, we found that the addition of doxorubicin led to p53 induction in NCI-H1299 cells. These results implied that, contrary to the tumor suppressive effect of p53 originally expressed in cells, doxorubicin-induced p53 probably contributed to drug resistance in lung cancer cells. In addition, coimmunoprecipitation assay showed that TopBP1 mediated the doxorubicin-induced p53 expression in NCI-H1299 cells, but not in NCI-H358, A549, and HCC827 cells.

\section{Conclusion}

In conclusion, our study for the first time demonstrated that TopBP1 is a target whose inhibition restores the sensitivity to doxorubicin in p53-null tumor cells. Furthermore, TopBP1 contributes to the drug resistance though regulation of doxorubicin-induced p53. Therefore, targeting TopBP1, in combination with chemotherapy, may represent a novel strategy for the treatment of chemotherapy-resistant tumors.

\section{Acknowledgment}

This research was supported by the Science Technology Department of Zhejiang Province Welfare projects: role and mechanism of TopBP1 on chemotherapy resistance in non-small cell lung cancer (2013C33100), the National 973 Basic Research Program of China (2013CB911303), Zhejiang Natural Science Foundation: identification and application of new molecular targets phlpp2 of lung cancer (Y15H160213), National Natural Science Foundation of China (81200662), and the Zhejiang Natural Science Foundation (LY14H120004).

\section{Disclosure}

The authors report no conflicts of interest in this work.

\section{References}

1. Siegel R, Ma J, Zou Z, Jemal A. Cancer statistics, 2014. CA Cancer J Clin. 2014;64(1):9-29.

2. Wang YY, Lin SX, Yang GQ, Liu HC, Sun DN, Wang YS. Clinical efficacy of CyberKnife combined with chemotherapy and hyperthermia for advanced non-small cell lung cancer. Mol Clin Oncol. 2013;1(3): 527-530.

3. Imai H, Shukuya $\mathrm{T}$, Yoshino R, et al. Efficacy and safety of platinum combination chemotherapy re-challenge for relapsed patients with non-small-cell lung cancer after postoperative adjuvant chemotherapy of cisplatin plus vinorelbine. Chemotherapy. 2013;59(4): 307-313.

4. Perol M, Chouaid C, Perol D, et al. Randomized, phase III study of gemcitabine or erlotinib maintenance therapy versus observation, with predefined second-line treatment, after cisplatin-gemcitabine induction chemotherapy in advanced non-small-cell lung cancer. $J$ Clin Oncol. 2012;30(28):3516-3524.

5. Ohashi R, Takahashi F, Cui R, et al. Interaction between CD44 and hyaluronate induces chemoresistance in non-small cell lung cancer cell. Cancer Lett. 2007;252(2):225-234.

6. Yang H, Wang W, Zhang Y, et al. The role of NF-E2-related factor 2 in predicting chemoresistance and prognosis in advanced non-small-cell lung cancer. Clin Lung Cancer. 2011;12(3):166-171.

7. Pallis AG, Syrigos KN. Epidermal growth factor receptor tyrosine kinase inhibitors in the treatment of NSCLC. Lung Cancer. 2013;80(2): 120-130.

8. Wright RH, Dornan ES, Donaldson MM, Morgan IM. TopBP1 contains a transcriptional activation domain suppressed by two adjacent BRCT domains. Biochem J. 2006;400(3):573-582.

9. Yamane K, Kawabata M, Tsuruo T. A DNA-topoisomerase-II-binding protein with eight repeating regions similar to DNA-repair enzymes and to a cell-cycle regulator. Eur J Biochem. 1997;250(3):794-799.

10. Rappas M, Oliver AW, Pearl LH. Structure and function of the Rad9binding region of the DNA-damage checkpoint adaptor TopBP1. Nucleic Acids Res. 2011;39(1):313-324.

11. Garcia V, Furuya K, Carr AM. Identification and functional analysis of TopBP1 and its homologs. DNA Repair (Amst). 2005;4(11): 1227-1239.

12. Kim JE, McAvoy SA, Smith DI, Chen J. Human TopBP1 ensures genome integrity during normal S phase. Mol Cell Biol. 2005;25(24): 10907-10915.

13. Yamane K, Tsuruo T. Conserved BRCT regions of TopBP1 and of the tumor suppressor BRCA1 bind strand breaks and termini of DNA. Oncogene. 1999;18(37):5194-5203.

14. Liu K, Bellam N, Lin HY, et al. Regulation of p53 by TopBP1: a potential mechanism for p53 inactivation in cancer. Mol Cell Biol. 2009;29(10):2673-2693.

15. Liu K, Luo Y, Lin FT, Lin WC. TopBP1 recruits Brg1/Brm to repress E2F1-induced apoptosis, a novel pRb-independent and E2F1-specific control for cell survival. Genes Dev. 2004;18(6):673-686.

16. Liu K, Lin FT, Ruppert JM, Lin WC. Regulation of E2F1 by BRCT domain-containing protein TopBP1. Mol Cell Biol. 2003;23(9): 3287-3304.

17. Herold S, Wanzel M, Beuger V, et al. Negative regulation of the mammalian UV response by Myc through association with Miz-1. Mol Cell. 2002;10(3):509-521.

18. Cescutti R, Negrini S, Kohzaki M, Halazonetis TD. TopBP1 functions with 53BP1 in the G1 DNA damage checkpoint. EMBOJ. 2010;29(21): 3723-3732.

19. Imbriano C, Gnesutta N, Mantovani R. The NF-Y/p53 liaison: well beyond repression. Biochim Biophys Acta. 2011;1825(2):131-139. 
20. Zhang W, Feng M, Zheng G, et al. Chemoresistance to 5-fluorouracil induces epithelial-mesenchymal transition via up-regulation of Snail in MCF7 human breast cancer cells. Biochem Biophys Res Commun. 2012;417(2):679-685.

21. Wu F, Li J, Jang C, Wang J, Xiong J. The role of Axl in drug resistance and epithelial-to-mesenchymal transition of non-small cell lung carcinoma. Int J Clin Exp Pathol. 2014;7(10):6653-6661.

22. Bang SW, Ko MJ, Kang S, et al. Human TopBP1 localization to the mitotic centrosome mediates mitotic progression. Exp Cell Res. 2011; 317(7):994-1004.

23. Jeon Y, Ko E, Lee KY, et al. TopBP1 deficiency causes an early embryonic lethality and induces cellular senescence in primary cells. J Biol Chem. 2011;286(7):5414-5422.

24. Choi SH, Yang H, Lee SH, Ki JH, Nam DH, Yoo HY. TopBP1 and Claspin contribute to the radioresistance of lung cancer brain metastases. Mol Cancer. 2014;13:211.

25. Manfredi JJ. The Mdm2-p53 relationship evolves: Mdm2 swings both ways as an oncogene and a tumor suppressor. Genes Dev. 2010;24(15): 1580-1589.

26. Marte B. Cancer: super p53. Nature. 2002;420(6913):279.
27. Riley T, Sontag E, Chen P, Levine A. Transcriptional control of human p53-regulated genes. Nat Rev Mol Cell Biol. 2008;9(5):402-412.

28. Garcia-Cao I, Garcia-Cao M, Martin-Caballero J, et al. "Super p53" mice exhibit enhanced DNA damage response, are tumor resistant and age normally. EMBO J. 2002;21(22):6225-6235.

29. Vogelstein B, Lane D, Levine AJ. Surfing the p53 network. Nature. 2000;408(6810):307-310.

30. Soussi T, Beroud C. Assessing TP53 status in human tumours to evaluate clinical outcome. Nat Rev Cancer. 2001;1(3):233-240.

31. Papadogianni D, Soulitzis N, Delakas D, Spandidos DA. Expression of p53 family genes in urinary bladder cancer: correlation with disease aggressiveness and recurrence. Tumour Biol. 2014;35(3):2481-2489.

32. Shetzer Y, Solomon H, Koifman G, Molchadsky A, Horesh S, Rotter V. The paradigm of mutant p53-expressing cancer stem cells and drug resistance. Carcinogenesis. 2014;35(6):1196-1208.

33. Kim CW, Lu JN, Go SI, et al. p53 restoration can overcome cisplatin resistance through inhibition of Akt as well as induction of Bax. Int J Oncol. 2013;43(5):1495-1502. 


\section{Supplementary materials}

$$
\rightarrow \text { Control } \rightarrow \text { p53 siRNA }
$$

A
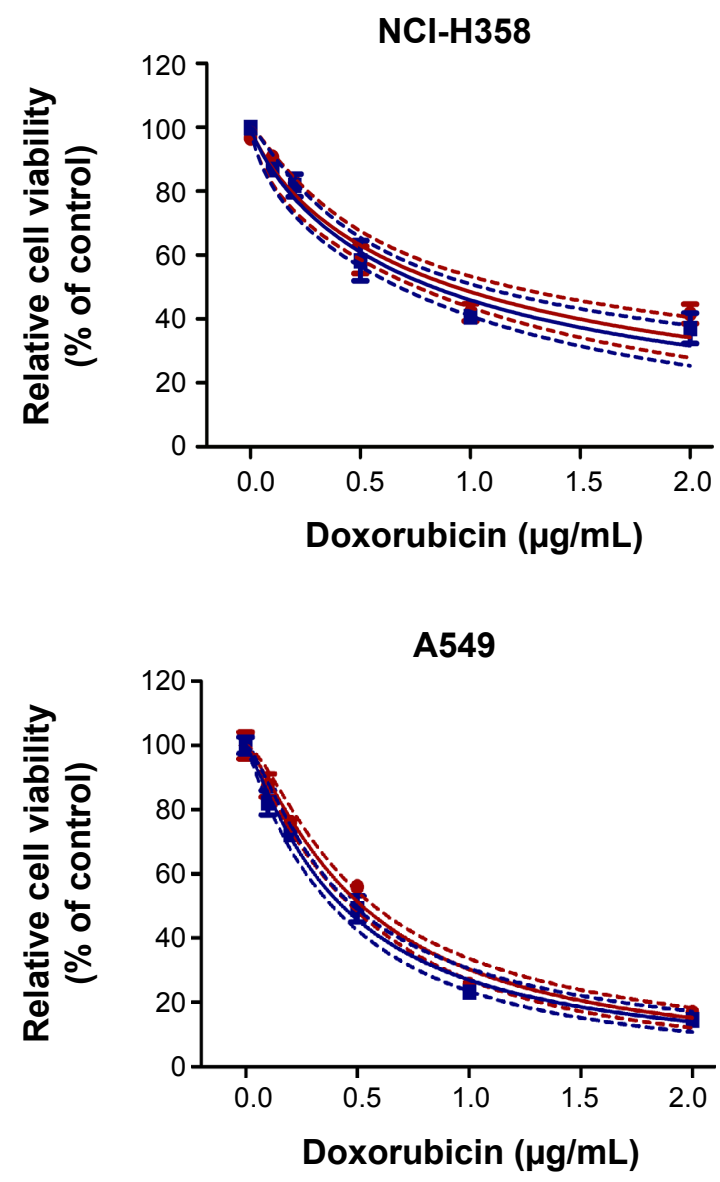
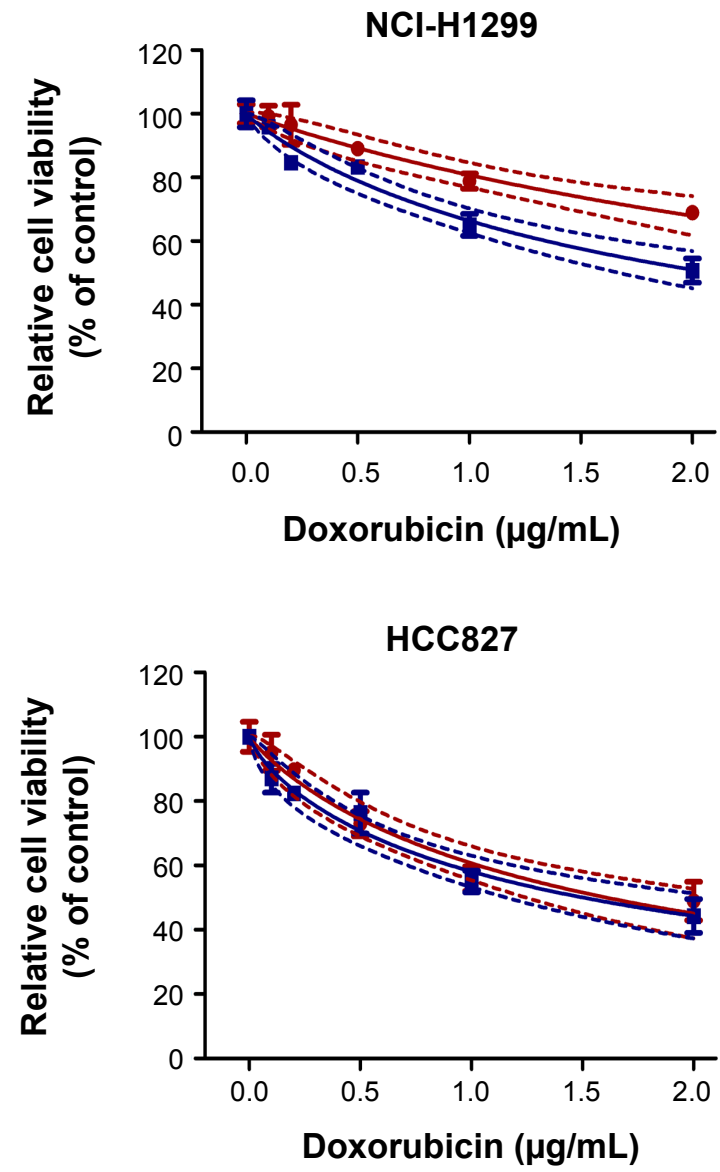

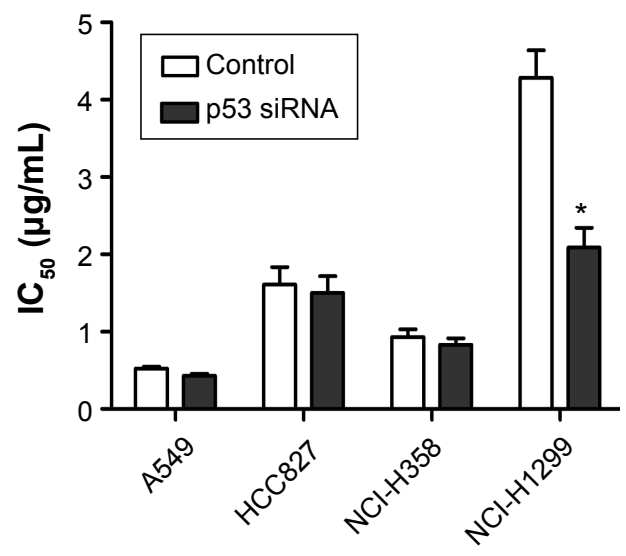

Figure SI (Continued) 

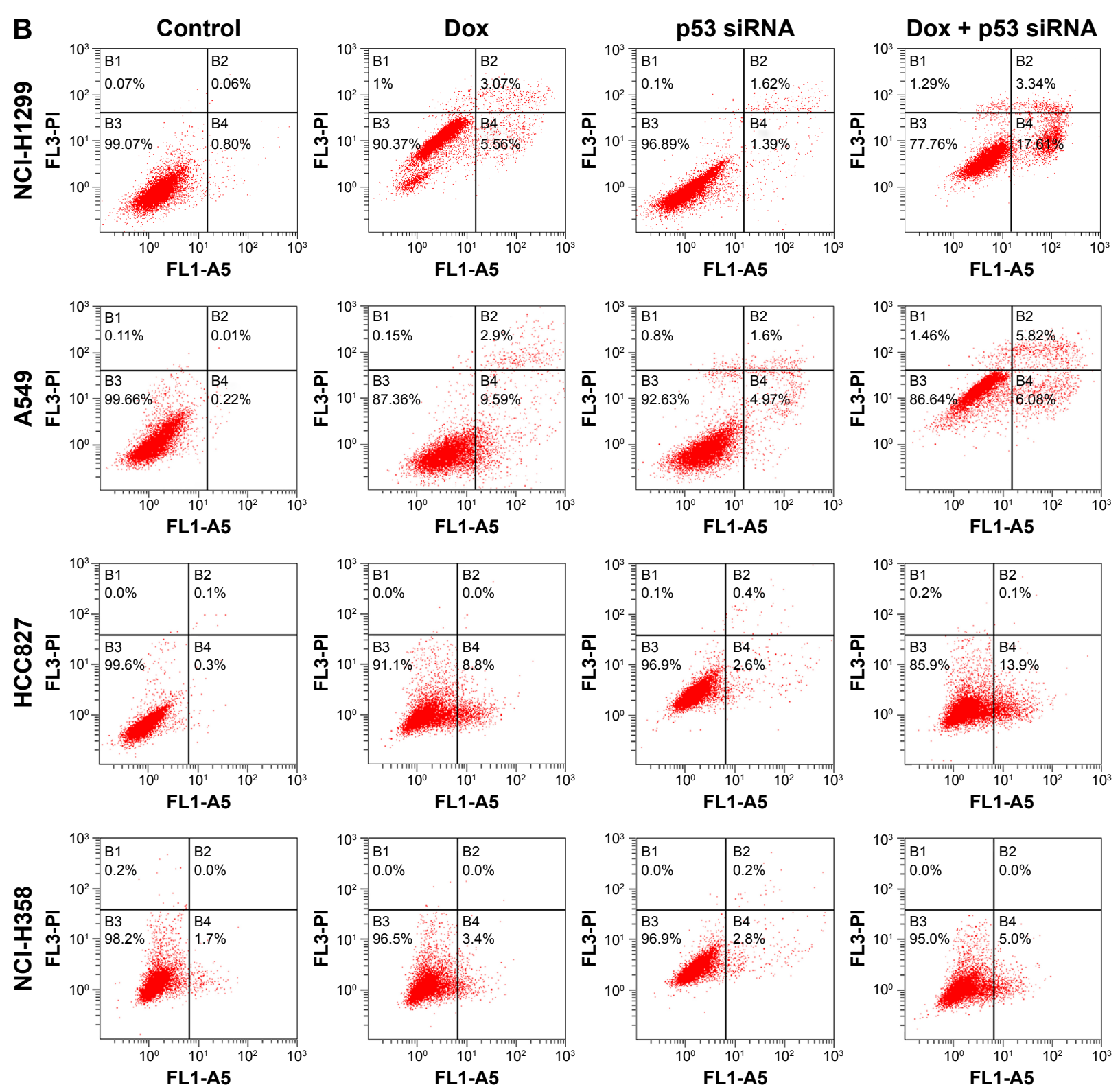

Figure SI Cell viability $(\mathbf{A})$ and apoptosis $(\mathbf{B})$ in doxorubicin (Dox)-treated non-small cell lung cancer cells before and after $\mathrm{p} 53$ knockdown. * represents statistical significance with $P<0.05$.

Abbreviations: siRNA, small interfering RNA; $I_{50}, 50 \%$ inhibitory concentration. 

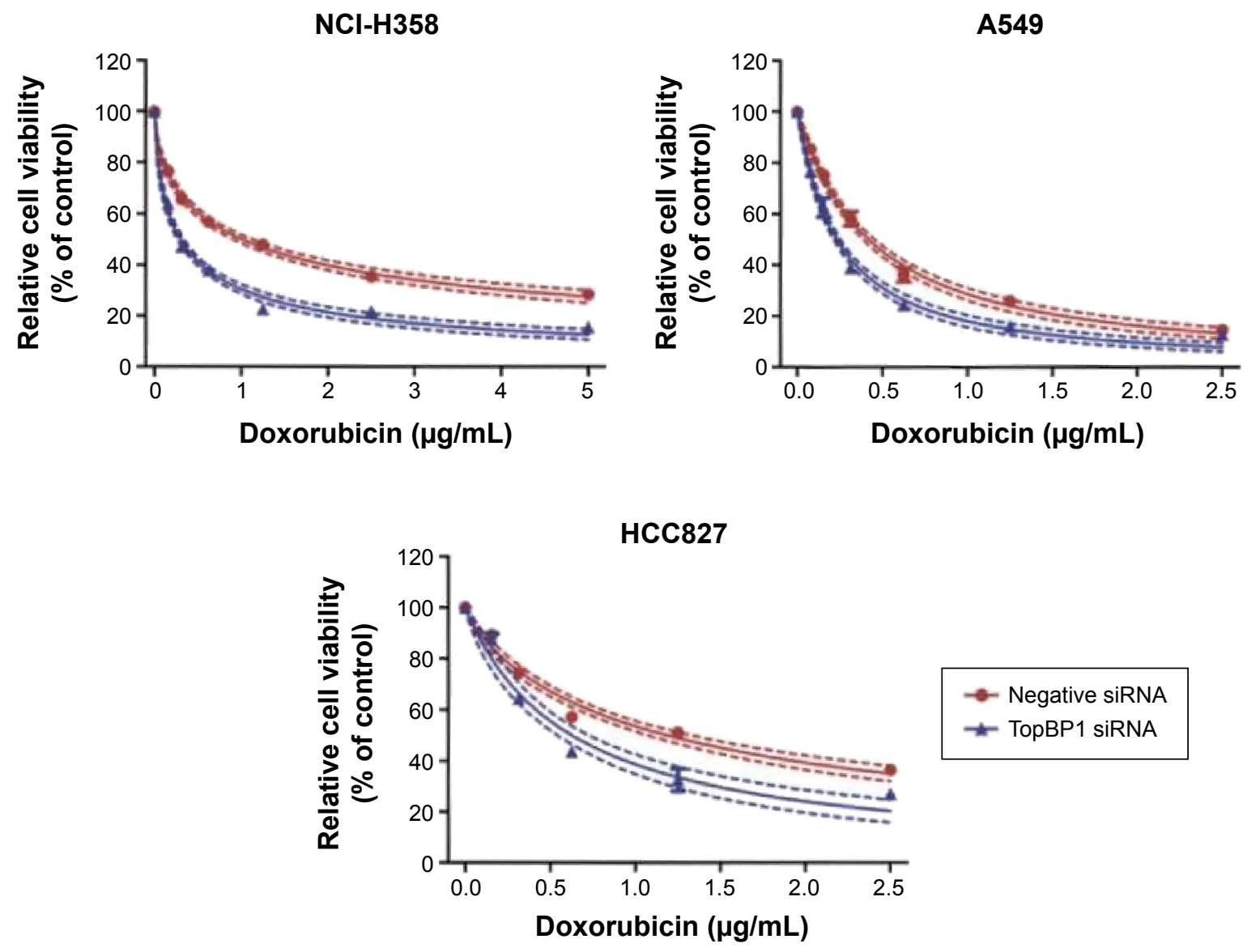

Figure S2 Effect of TopBPI in non-small cell lung cancer cells.

Abbreviations: TopBPI, topoisomerase II $\beta$ binding protein I; siRNA, small interfering RNA.

\section{Publish your work in this journal}

Drug Design, Development and Therapy is an international, peerreviewed open-access journal that spans the spectrum of drug design and development through to clinical applications. Clinical outcomes, patient safety, and programs for the development and effective, safe, and sustained use of medicines are the features of the journal, which has also been accepted for indexing on PubMed Central. The manuscript management system is completely online and includes a very quick and fair peer-review system, which is all easy to use. Visit http://www.dovepress.com/testimonials.php to read real quotes from published authors 